\title{
SoLid: Search for Oscillations with a Lithium-6 Detector at the SCK • CEN BR2 reactor
}

\author{
Daniel Saunders* \\ University of Bristol \\ E-mail: dan.saundersabristol.ac.uk
}

\begin{abstract}
The disappearance of reactor antineutrinos into a new neutral state (called sterile neutrino) has been proposed as a possible explanation for the recent reactor and Gallium anomalies arising from the re-analysis of reactor flux and calibration data of previous neutrino experiments. A way to test this hypothesis is to look for distortions of the anti-neutrino energy caused by oscillation from active to sterile neutrino at several close stand-off distances $(\sim 5.5-10 \mathrm{~m})$ from a compact reactor core. Due to the small anti-neutrino cross-section, the main challenge in achieving a precise measurement is to control the high level of gamma rays and neutron background that are present at close stand off and to the surface.

The SoLid experiment intends to search for active-to-sterile anti-neutrino oscillation at very short baseline of the SCK • CEN BR2 research reactor. A novel approach to measuring reactor antineutrinos was developed based on an innovative sandwich of composite polyvynil-toluene and ${ }^{6} \mathrm{LiF}: \mathrm{ZnS}$ scintillators. The system is highly segmented and read out by a network of wavelength shifting fibers and MPPCs. High experimental sensitivity can be achieved with the combination of high granularity, high neutron-gamma discrimination using ${ }^{6} \mathrm{LiF}: \mathrm{ZnS}(\mathrm{Ag})$ scintillator and precise localisation of the inverse beta decay products. We will describe the principle of detection, the detector design and we will focus on the performance of the full scale SoLid prototype module 1 installed at BR2 early 2015 demonstrating the technology. We will present first results on selecting inverse beta decay events using the first data set collected with the prototype module in 2015, and will show the physics reach of the next phase that will start data taking in early 2017. Some design optimisations for the next phase will also be presented.
\end{abstract}

38th International Conference on High Energy Physics

3-10 August 2016

Chicago, USA

\footnotetext{
*Speaker.

${ }^{\dagger}$ on behalf of the SoLid Collaboration
} 


\section{Introduction}

The reactor anti-neutrino anomaly [1] and the gallium anomaly [2] both arise due to a deficit of electron anti-neutrinos, measured by detectors at a distance $\sim 100 \mathrm{~m}$ from nuclear reactors or intense radioactive sources. The SoLid collaboration aim to determine whether these anomalies may be due to a very short baseline oscillation into a new sterile neutrino flavour. The experiment will search for an oscillation by measuring the anti-neutrino energy spectrum at a range of distances from the BR2 reactor core at $\mathrm{SCK} \bullet \mathrm{CEN}$. Being a research reactor, the small reactor core diameter of $0.5 \mathrm{~m}$ is ideal for the measurement, allowing the core to be modelled as a point source with minimal smearing.

The fuel composition is highly enriched Uranium, and measurements of the neutrino energy spectrum offer further handles in understanding the observed distortion of the spectrum in the region around $5 \mathrm{MeV}$ reported by previous experiments [3].

Deploying a tonne scale detector at ground level, next to a nuclear reactor is particularly challenging due to the high rate of cosmic rays and reactor related background events. To combat these backgrounds, the SoLid collaboration have devel-

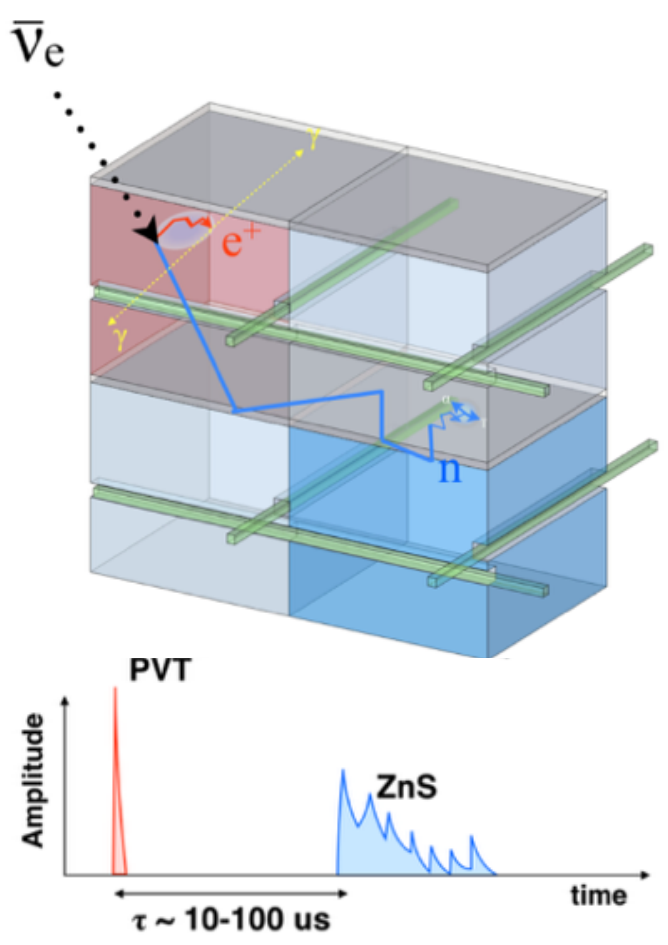

Figure 1: SoLid detection principle. The antineutrino decays into a positron, which gives a scintillation signal from the PVT, and a neutron, which captures on the Lithium-6 scintillator. The positron and neutron are correlated in both space and time. oped a novel and highly segmented technology for the detection of inverse beta decay interactions (IBD) based on a composite scintillator. The additional topological information offers new and unexplored handles for characterising neutrino interactions, allowing better separation of signal and background. The entire detector is composed of thousands of small $(5 \mathrm{~cm})$ cubical cells. The bulk of each cell is polyvinyl toluene (PVT), which scintillates at the timescale of a few nanoseconds for traversing charged particles, such as positrons and muons. Each cell is lined with two sheets of ${ }^{6} \mathrm{LiF}: \mathrm{ZnS}$, which scintillates upon neutron capture. The light from the cubes is read-out via wavelength shifting fibres that are placed along four (two in the case of the prototype) grooves that are cut in perpendicular sides of the cubes, allowing the position of the cube(s) to be reconstructed. The time and position of the positron and neutron are correlated, as is demonstrated in figure 1. Silicon photomultipliers (SiPMs) are attached to the end of each fibre, and a mirror placed at the opposite end to increase the light yield. The emission process of the ${ }^{6} \mathrm{LiF}: \mathrm{ZnS}$ (see [6]) gives rise to a characteristically different scintillation signal compared to the PVT. The signal is spread over a period of a few microseconds, and often contains multiple emission peaks, and can be effectively separated from the large number of EM events (see [7]). 


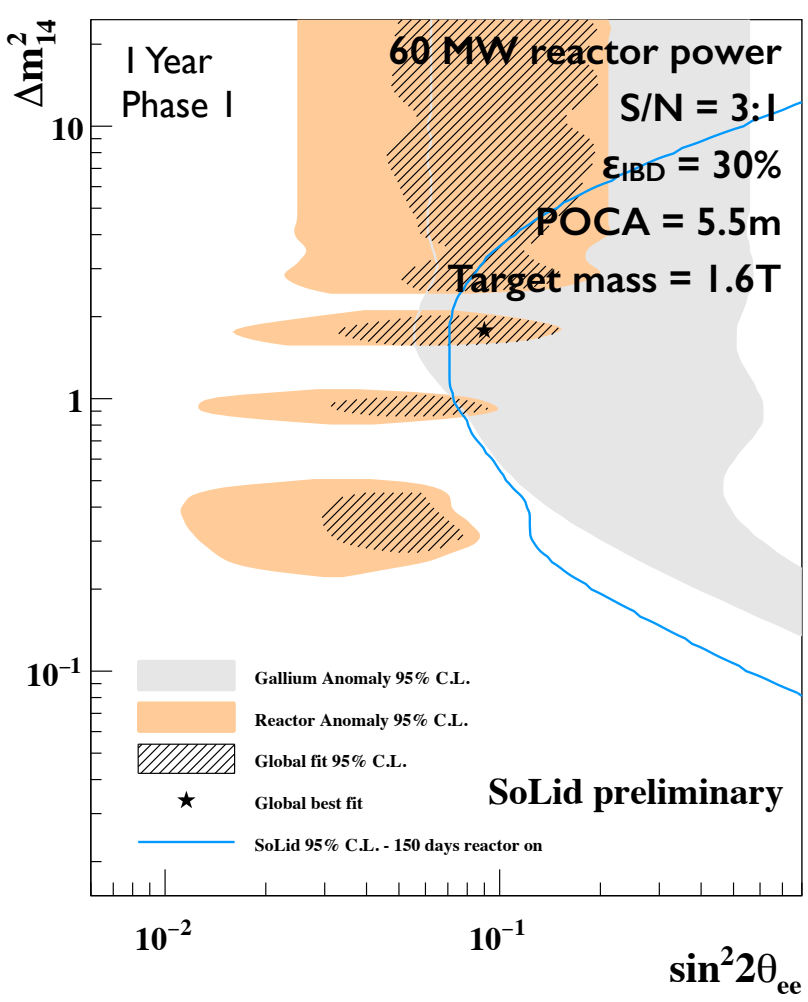

Figure 2: Phase 1 sensitivity after approximately one year of data taking.

ity is shown in figure 2 (as in [8]).
A large scale prototype detector, composed of $16 \times 16 \times 9$ cubes, was constructed and commissioned in early 2015. This prototype had minimal passive shielding, and a simple threshold trigger for both EM and neutrons signals. Due to the large reactor EM background, a high trigger threshold was required to achieve a manageable data rate, resulting in a reduced neutron trigger efficiency. The detector took a short 3 day reactor on run and many months of reactor off and source data. Although the reactor on sample is too small to be used for neutrino studies, the reactor off sample is suitable for many background studies, demonstrating the power of the segmented design.

The next phase of the experiment, which will perform an oscillation search at the reactor, is currently being built, and is composed of 50-60 planes. This paper presents various analysis results from the large scale prototype, with emphasis on the improvements for the phase 1 design. The expected sensitiv-

\section{Prototype Results}

The MPPCs output data in the form of digitised waveforms 256 samples long (see [6] and [7] for examples). Channels that trigger within a time co-incidence of $50 \mathrm{~ns}$ are grouped together to form events, so simultaneous signals from the detector form a single event. IBD interactions are therefore formed of two events.

For particle identification, it is crucial to separates neutrons from low energy EM events and to efficiently tag muons. Effective muon separation is critical to reduce muon-related backgrounds, such as muon spallation within the detector, and to reduce the accidental backgrounds. Muons can be very effectively identified due to their large amplitude signals and high channel multiplicity compared to other events such as positrons [4]. Neutron identification is performed using pulse shape discrimination. The fact that ${ }^{6} \mathrm{LiF}: \mathrm{ZnS}$ emission of scintillation light is significantly slower compared to EM signals, the integral divided by the amplitude of waveforms can effectively separate the two categories of signal, resulting in negligible contamination for the prototype configuration [7]. 

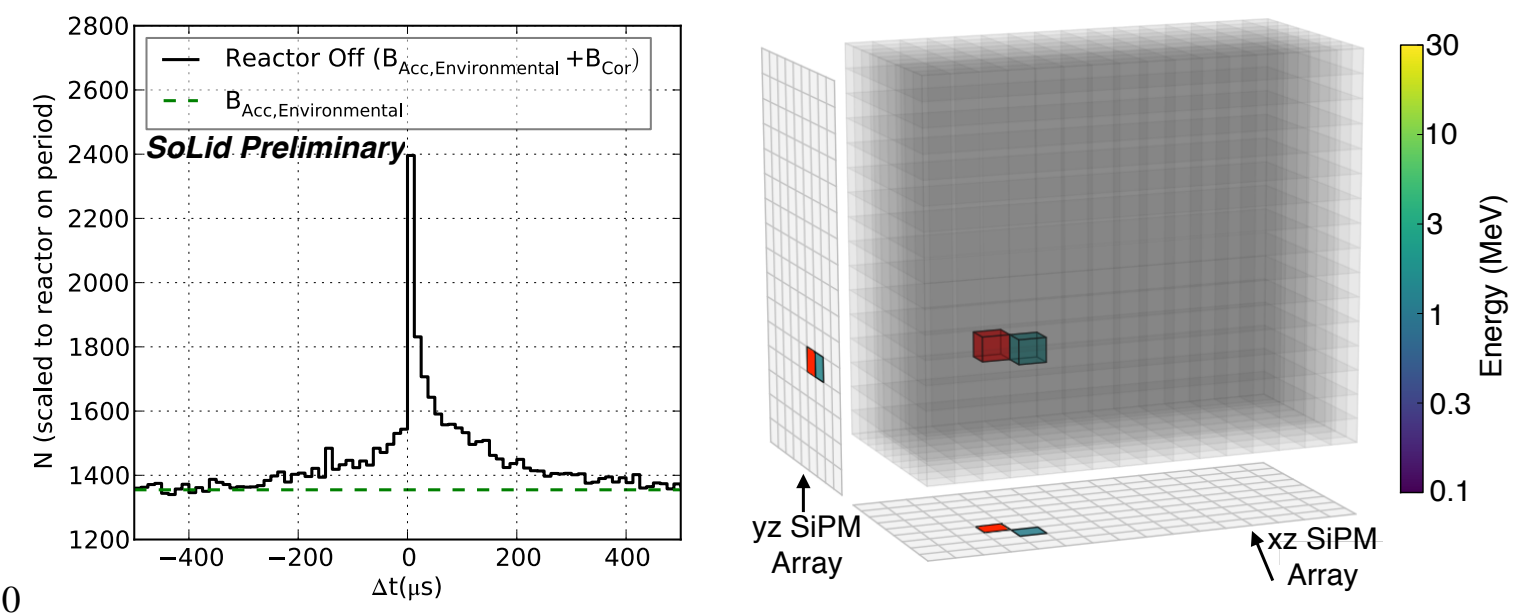

Figure 3: Left: time correlation between neutron and positron candidates (prior to other selections). Right: IBD candidate example.

IBD candidates are found by searching for time coincidences between neutrons and EM events at the neutron thermalisation timescale. This correlation is shown in the left of figure 3 , which shows the time separation between neutron and EM events $\Delta t$, prior to any other analysis selections for reactor off data. A correlated component is observed at the scale $\sim 100 \mu \mathrm{s}$, corresponding to the Correlated Background - cases where the neutron and positron originate from the same interaction, and are correlated in time at the neutron thermalisation time scale. This sits atop a large flatter background, which corresponds to the Accidental Background - random mismatches of EM events (e.g from the reactor reactor) and neutron events (e.g thermal cosmics).

The selection cut $0<\Delta \mathrm{t}(\mu \mathrm{s})<220$ is used to initially form IBD candidates, set to include $90 \%$ of correlated candidates (and therefore signal). An example is shown in the event display shown in the right of figure 3. For each candidate, several features are extracted such as positron multiplicity, radial separation, and positron energy. Additional analysis cuts are placed on the IBD features to reduce the backgrounds and increase the signal to noise ratio [9]. Of these, it is found the topological selections, such as radial separation and channel multiplicity, are extremely powerful for background rejection. It can be shown that the correlated background can be reduced by around a factor of twenty, and the accidental background is reduced by over two orders of magnitude [9], [5].

\subsection{Reactor On/Off Comparison}

The prompt energy distribution of IBD candidates for the reactor on period is shown in figure 4. The predicted background model is also superposed. This is the sum of the correlated background, whose rate is assumed to be fixed for the reactor on and off periods, and the reactor on accidental background, found using shifted time windows. Good agreement is observed between the background prediction and the measurement, suggesting the background model is valid. There is no significant excess in events corresponding to neutrinos, as expected, given the small number of candidates (recall the prototype had minimal passive shielding and low neutron efficiency due to a simple threshold trigger). 


\section{Selection of Phase 1 Optimisations}

\subsection{Passive Shielding}

To further reduce the backgrounds for phase 1 of the experiment, $50 \mathrm{~cm}$ of water shielding will be placed as walls surrounding the entire detector, and slabs of polyethylene will be used to shield the top of the detector. This type of shielding specifically targets the correlated background due to cosmic neutrons and cosmic muon spallation, and simulation studies using cosmic generators predict a reduction by in the rate of highly energetic neutrons (1-20 MeV) of around a factor 10. A schematic of the detector with this shielding is shown in figure 5 .

\subsection{Neutron Trigger}

The prototype triggering system required two MPPCs to be above threshold and time coincident in a single detector plane for both neutron and EM signals. Due to the large EM reactor background, the threshold had to be placed high to avoid DAQ saturation, resulting in a low neutron efficiency. This is rectified in phase 1 by the use of a dedicated neutron

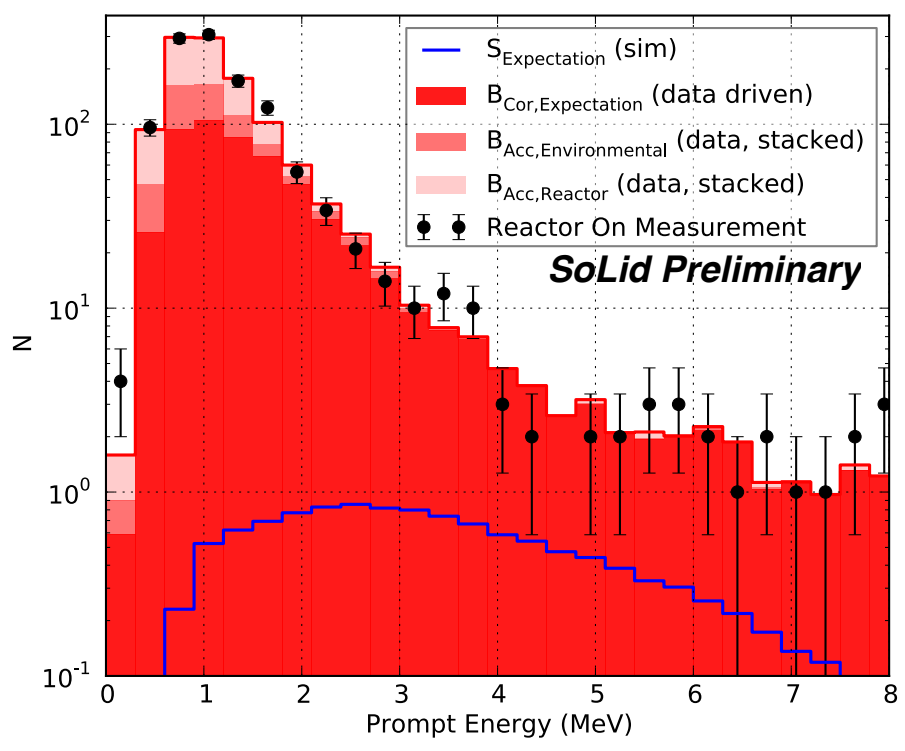

Figure 4: Prompt energy distribution for reactor on data. The predicted background is shown in red (stacked). The small predicted neutrino contribution is shown in blue. Note that the prototype module trigger threshold was set around $500 \mathrm{keV}$.

trigger. By using discriminating variables that better separate neutrons and EM signals, greater efficiency can be achieved, as seen in figure 6. By comparing the threshold requirement with a discriminating variable such as time-over-threshold, a significantly greater efficiency is possible for the equivalent rate of false positives.

Following a neutron trigger, all data from \pm 2 planes either side of the neutron signal location for the previous $\sim 500 \mu \mathrm{s}$ can be recorded (zero suppressed); the efficiency for detecting signals from the prompt event (e.g positron, proton recoil) is thus very high. Further, it is found that the dominant contaminant of false positives passing this neutron trigger (and entering the buffer) arise 


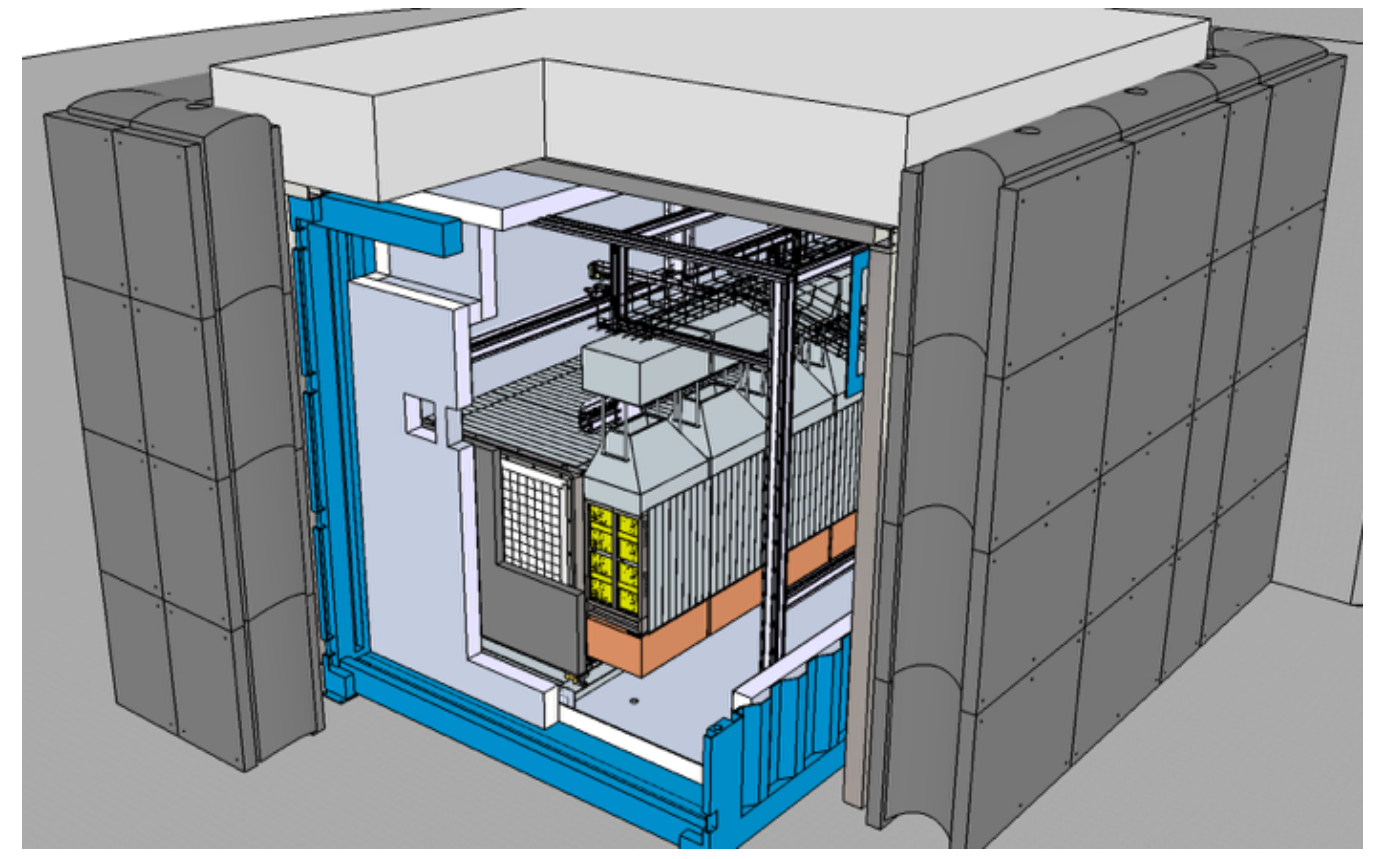

Figure 5: Schematic of phase 1 of the experiment with passive shielding.

from the dark count of the MPPCs. For this reason, and better stability, the entire detector will be cooled to around 5 degrees Celsius, reducing the dark count rate by over an order of magnitude.

\section{Summary}

The SoLid experiment will search for neutrino oscillations at a very short baseline of $\sim 6-8 \mathrm{~m}$ from the BR2 nuclear reactor. A novel highly segmented technology has been developed to perform the measurement, offering new handles to reduce the large backgrounds present when performing measurements on the surface and close to a reactor core. The technology has been demonstrated using a large scale prototype that was commissioned in early 2015. The segmented design has been shown to give significant gains in background reduction, reducing both accidental and correlated backgrounds by orders of magnitude. Based on experience gained from this prototype, the next phase of the experiment has been optimised and is currently being constructed. First data is expected early 2017 , and the expected sensitivity of the .

\section{References}

[1] Mention, G. et al., Reactor antineutrino anomaly, Phys. Rev. D 83, 073006 (2011)

[2] Giunti, C. et al., Statistical significance of the gallium anomaly, Phys. Rev. C 83, 065504 (2011)

[3] Patrick Huber, Reactor antineutrino fluxes - status and challenges, Neutrino 2016.

[4] Daniel Saunders, Muon Calibration at SoLid, EPS 2015.

[5] Daniel Saunders, Inverse Beta Decay Signatures at SoLid, Neutrino 2016. 


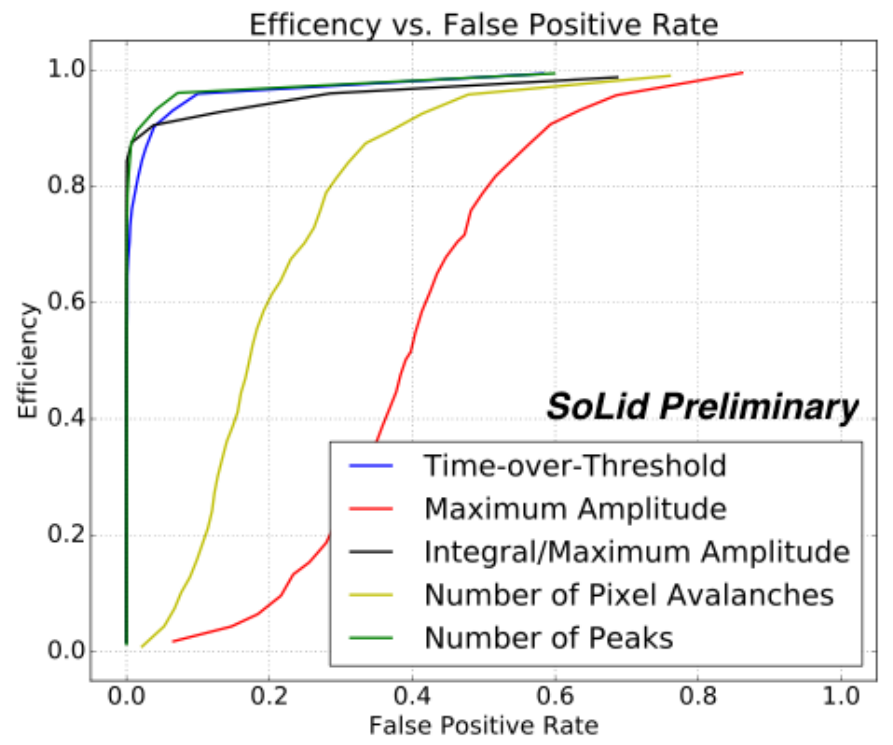

Figure 6: ROC curves for potential phase 1 neutron trigger algorithms using an AmBe source.

[6] Simon Vercaemer, Neutron identification in the SoLid experiment, EPS 2015.

[7] Leonidas Kalousis, SoLid: A compact neutrino detector for very short-baseline neutrino experiments, these proceedings.

[8] Leonidas Kalousis, The SoLid experiment, Neutrino 2016.

[9] Ianthe Michiels, Overview of the background reduction techniques applied in the SoLid experiment, these proceedings. 